\title{
The correlation between near vision and smartphone use among ageing populations
}

\author{
Qinrui $\mathrm{Hu}^{1,2,3 \#}$, Yang $\mathrm{Li}^{1,2,3 \#}$, Bin Wang ${ }^{1,2}$, Xueying Qin ${ }^{3}$, Tao Ren ${ }^{3}$, Yonghua $\mathrm{Hu}^{3}$, Xiaoxin $\mathrm{Li}^{1,2}$ \\ ${ }^{1}$ Fujian Provincial Key Laboratory of Ocular Surface and Corneal Diseases, Xiamen, China; ${ }^{2}$ Eye Institute and Affiliated Xiamen Eye Center of \\ Xiamen University, School of Medicine, Xiamen University, Xiamen, China; ${ }^{3}$ Department of Epidemiology and Biostatistics, School of Public \\ Health, Peking University Health Science Centre, Beijing, China \\ Contributions: (I) Conception and design: Q Hu, Y Li, X Li, Y Hu; (II) Administrative support: Q Hu ,Y Li, B Wang; (III) Provision of study materials \\ or patients: Q Hu and Yang Li; (IV) Collection and assembly of data: Q Hu, Y Li; (V) Data analysis and interpretation: Q Hu, Y Li, X Qin, T Ren; (VI) \\ Manuscript writing: All authors; (VII) Final approval of manuscript: All authors. \\ "These authors contributed equally to this work and should be considered as co-first authors. \\ Correspondence to: Xiaoxin Li. Xiamen Eye Center affiliated with Xiamen University, Xiahe Road 336, Siming District, Xiamen, China; Email: \\ drlixiaoxin@163.com; Yonghua Hu. Department of Epidemiology and Biostatistics, School of Public Health, Peking University Health Science \\ Centre, Xueyuan Road 38, Haidian District, Beijing, China. Email: yhhu@bjmu.edu.cn.
}

Background: This population-based, cross-sectional study aimed to assess the correlation between near
vision and smartphone usage among people aged $\geq 50$ years in China.
Methods: This study was performed on the ocular health status of residents in Fujian Province, Southeast
China. People aged $\geq 50$ years were recruited. The main contents of the survey for the residents included
socioeconomic status, uncorrected near visual acuity (UNVA), refractive state, as well as a questionnaire
about smartphone usage and visual quality.
Results: Smartphone adoption accounted for $67.7 \%(4,702$ individuals) of the total population, which
decreased with age in the elderly. Smartphone owners had a higher average UNVA of $0.31 \pm 0.18$ than non-
smartphone owners $(0.23 \pm 0.14)$, and the difference was statistically significant (P<0.001). Individuals who
used smartphones for a long time usually had better UNVA (Pearson correlation coefficient $0.144, \mathrm{P}<0.001)$.
The rates of complaints of distance vision loss, near vision loss, ocular surface discomfort in smartphone
users were significantly higher than that of non-users $(\mathrm{P}<0.001)$. Also, smartphone adoption and visual
quality differed between urban and rural respondents.

Conclusions: Smartphone adoption and usage time in the elderly significantly decreased with age and UNVA, and the performance of visual impairment was not consistent in urban and rural areas.

Keywords: Smartphone; near vision; ageing; China; cross-sectional study

Submitted Nov 25, 2021. Accepted for publication Jan 11, 2022.

doi: 10.21037/apm-21-3830

View this article at: https://dx.doi.org/10.21037/apm-21-3830

\section{Introduction}

Smartphones are permeating numerous aspects of our lives, and have provided revolutionary reforms to society (1). In 2018, the scale of mobile payment reached 34 trillion dollars in China, and smartphone applications-scan payments infiltrated every aspect of our social activities, from hospitals to stalls (2). However, the widespread use of mobile phones also leads to some problems in society, especially for the elderly. Firstly, the near vision function of elderly individuals affects the wide application of mobile phones in these groups, as uncorrected near visual acuity (UNVA), which refers to the acuity that can be achieved at a near distance, is usually decreased in the elderly. Secondly, complex smartphone designs also contribute to the alienation of the elderly, as few apps are suitable for 
them currently. Many individuals are forced to confront this social marginalization by the intelligent applications on smartphones, and the Chinese government is considering legislating in favour of tilting intelligent design towards the ageing population.

On the other hand, the global demand for smart products is increasing among the elderly population. A study from the United States indicated that $37 \%$ of adults aged 60 years and over spent $\geq 5$ hours per day on digital devices (3). In the United Kingdom (UK), the use of technology grew rapidly between 2011 and 2017. The proportion of the population who were recent internet users more than doubled in those aged $\geq 75$ years compared to other age groups, and the proportion increased from $52.0 \%$ to $77.5 \%$ in those aged $65-74$ years (4). Entertainment, communication, and access to information are on the rise and available on smartphones, and the elderly are also eager to participate in social activities.

However, another serious problem associated with smartphone usage in the ageing population is the decreasing visual function, which differs from adolescents. The current state of the research is that, for adolescents, the public are afraid that they would be addicted to smartphones $(5,6)$. Longer smartphone use may increase the likelihood of ocular symptoms, including myopia, asthenopia, and ocular surface disease (7). The elderly may face the same problem, but few relevant literatures was presented. This is one of the issues we're intended to explore. As smartphones are primarily used as social communication tools for the new generation, in the case of the elderly, the society should concern that the elderly would be isolated gradually from society, for the decline in the usage of smart products. On smartphone usage, the elderly face distinctly different challenges: presbyopia and ageing. Different from myopia (do well in near vision), presbyopia leads to a decline in near-vision function, affecting the use and reading of smartphones in middle-aged and elderly people. Globally, functional presbyopia affects 666.7 million people aged 50 years or older (8), and among these people, many individuals spend no less time on smartphones than teenagers. These overlapping factors may directly affect the use of smartphones and communication in elderly individuals. The public should take the nearly 700 million elderly people worldwide into account when developing technology (9). For the foundation of prospective research and policies for smartphone usage, details regarding these populations are in urgent demand. Unfortunately, due to a previous lack of concern, relevant research on the elderly is currently limited.

In China, 765 million people utilize mobile payment, accounting for $85.3 \%$ of mobile users (1). Residents have been profoundly influenced by the influx of smartphones, and the trend has undoubtedly impacted the visual burden on elderly individuals. However, the evolution of this trend remains unknown. Therefore, this investigation aimed to explore the overall utilization of smartphones and its correlation with the near vision of people aged 50 years or older, and to provide data support for further research and policy-making. We present the following article in accordance with the SURGE reporting checklist (available at https://apm.amegroups.com/article/view/10.21037/apm$21-3830 / \mathrm{rc})$.

\section{Methods}

\section{Study design}

This study [known as the Fujian Eye Study (FJES)], a population-based, cross-sectional survey on the public ocular health status of Fujian Province, southeast China, was approved by the Ethics Committee of Xiamen Eye Center affiliated with Xiamen University (No. XMYKZXKY-2018-001), and was performed by Eye Institute and Affiliated Xiamen Eye Center of Xiamen University. The study was conducted in accordance with the Declaration of Helsinki (as revised in 2013). People aged $\geq 50$ years were selected for the study from 2018 to 2019, and informed consent was taken from all the participants. All investigators underwent unified training during the implementation of the project and examinations were performed consistently.

\section{Data collection and analysis}

The main contents of the survey were as follows general information (age, sex, race, etc.); the questionnaire (socioeconomic status; eye status; physical status; phone use; others); UNVA; refractive state; and slit-lamp examination. UNVA was measured at $30 \mathrm{~cm}$, using a logarithm of the minimum angle of resolution based on the NVA tumbling $\mathrm{E}$ chart. The values of the chart were $0,0.125$ (20/160), $0.16,0.20,0.25,0.32,0.4(20 / 50), 0.5,0.63$, and $0.8(20 / 25)$. Presbyopia was defined as vision below 0.4 (20/40). The main contents of the visual quality questionnaire were as follows: "Have you recently felt any ocular discomfort, such as itching, dryness, foreign body sensation and so on?", "Do you find it difficult to see distant objects, or have you experienced 
distance vision loss?", "Do you find it difficult to work or read in near work, or have you experienced near vision loss?", and "Do you bave a smartphone?". The options for answering these questions were yes or no. For the question "How much time do you use a smartphone per day on average?", the options were under half an hour, half an hour to an hour, 1 to 2 hours, 2 to 4 hours, 4 to 6 hours, and more than 6 hours.

\section{Statistical analysis}

Stata/SE statistical software (Stata for Windows, version 15.1, StataCorp LLC., Lakeway Drive, College Station, TX, USA) was used for statistical analysis in the study. Analysis of variance (ANOVA) was applied to compare the mean among groups of normally distributed parameters. The Chi-square $\left(\chi^{2}\right)$ test was used to compare the proportions. All $\mathrm{P}$ values less than 0.05 were considered statistically significant.

\section{Results}

A total of 8,211 individuals aged 50 years and older were enrolled in the FJES 2018-2019. Among these, 6,942 individuals $(84.5 \%)$ completed the questionnaire and were eventually included in this study. The mean age of the participants was $64.2 \pm 8.8$ years. The population was divided into seven subgroups by age. As there was no significant difference $(\mathrm{P}=0.829)$ in the mean UNVA for either eye among the participants, the right eye was considered the main object in this study.

\section{Adoption of smartphones}

Smartphone users accounted for $67.7 \%$ (4,702 individuals) of the total sample, and the rate of smartphone adoption in the population decreased with age (Table 1). In the 50-54-year age group, smartphone adoption was $87.9 \%$, which dropped to $32.3 \%$ in those over 80 years old. The differences among age groups in smartphone adoption were significant $(\mathrm{P}<0.001)$. Women had a higher level of smartphone adoption than men $(71.6 \%$ vs. $65.2 \%, \mathrm{P}<0.001)$, and adoption among urban respondents $(72.5 \%)$ was higher than that among rural respondents $(62.5 \%, \mathrm{P}<0.001$, Figure 1$)$.

\section{Time on smartphone}

In total, $57.4 \%$ of the respondents spent less than 1 hour on smartphones, and $34.7 \%$ spent less than 0.5 hours on smartphones. The time showed a steep decline with age. In the 50-54-year age group, $47.2 \%$ spent less than 1 hour per day on smartphones. In the 60-64-year group, the rate rose to $59.5 \%$. Urban respondents spent more time on smartphones than rural respondents $(\mathrm{P}<0.001)$. The average time spent on smartphones for the total sample was $1.33 \pm 1.33$ hours. For urban respondents (Figure 2), it was $1.42 \pm 1.39$ hours, while for rural respondents (Figure 3), it was $1.19 \pm 1.21$ hours, and the difference was statistically significant $(\mathrm{P}<0.001)$.

\section{UNVA}

Smartphone owners had a higher average UNVA $(0.31 \pm 0.18)$ than non-smartphone owners $(0.23 \pm 0.14)$, and the difference was statistically significant $(\mathrm{P}<0.001)$. The trends of UNVA in different age subgroups are shown in Figures 2,3. On the graph, there is a sharp decrease in UNVA from 50 to 65 years $(\mathrm{P}<0.001)$, whereas a relatively stable UNVA plateau forms from 65 to 80 years old. The time spent on smartphones and UNVA shows a linear correlation (Pearson correlation coefficient $0.144, \mathrm{P}<0.001$, Figure 4).

\section{Vision quality (complaints of distance vision loss, near vision loss, ocular surface discomfort)}

The rates of complaints of distance vision loss, near vision loss, ocular surface discomfort in smartphone users were significantly higher than that of non-users $(\mathrm{P}<0.001$, respectively). The trend of complaint rates by time is shown in Figure 5. With the prolonging of smartphone usage time, the rate of complaints about ocular surface discomfort increased significantly in both the urban and rural subgroups $(\mathrm{P}=0.022$ and $\mathrm{P}=0.043$, respectively). However, there was no statistically significant difference in the rate of complaints about distance vision loss $(\mathrm{P}=0.118$ and $\mathrm{P}=0.064$, respectively). The proportion of complaints about near vision loss among both urban and rural respondents showed an increasing trend with usage time $(\mathrm{P}=0.006$ and $\mathrm{P}=0.007$, respectively). In terms of visual quality, the complaint rates increased sharply after smartphone use over 2 hours among respondents. Generally, the rate of complaints was higher among urban respondents than among rural respondents $(\mathrm{P}<0.001)$.

\section{Discussion}

Our study provides a preliminary insight into the use of smartphones among elderly people. Both the popularity 


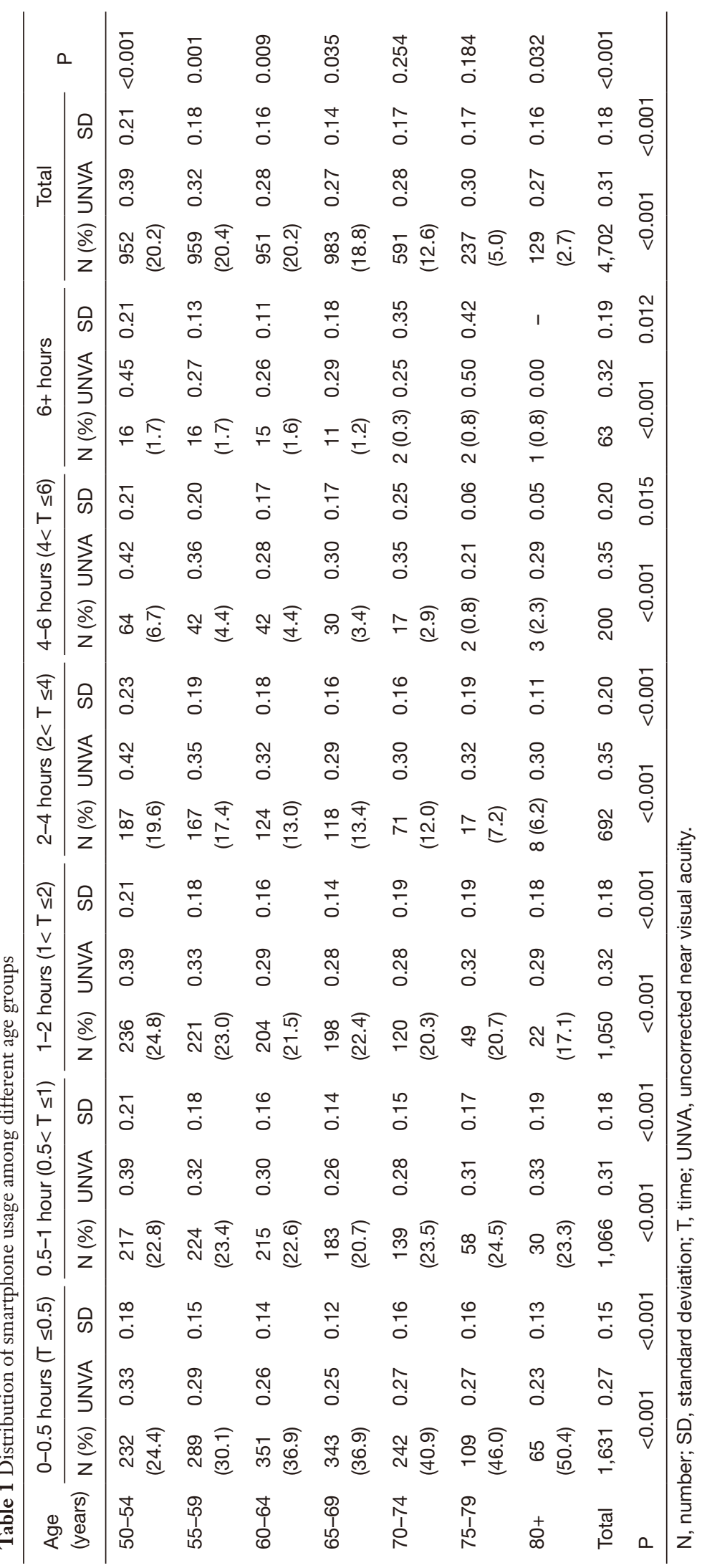




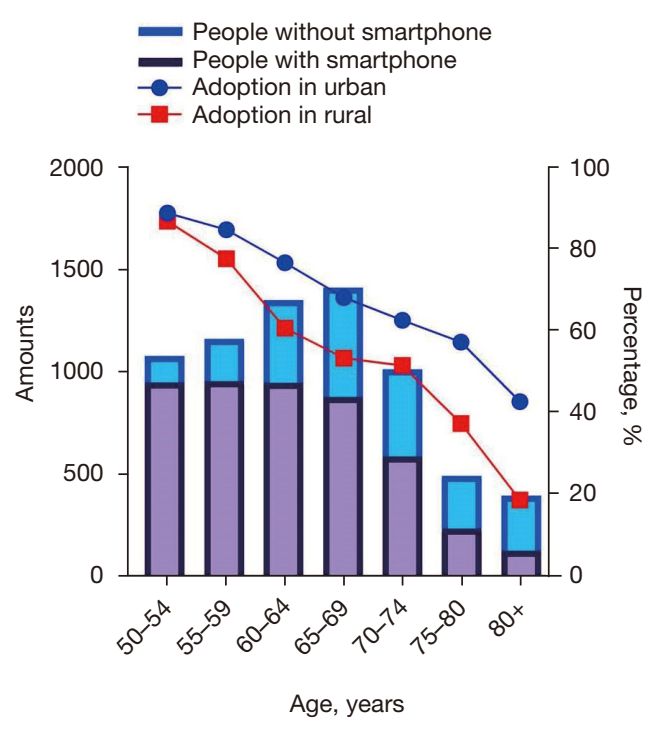

Figure 1 Amounts and rates of smartphone usage.

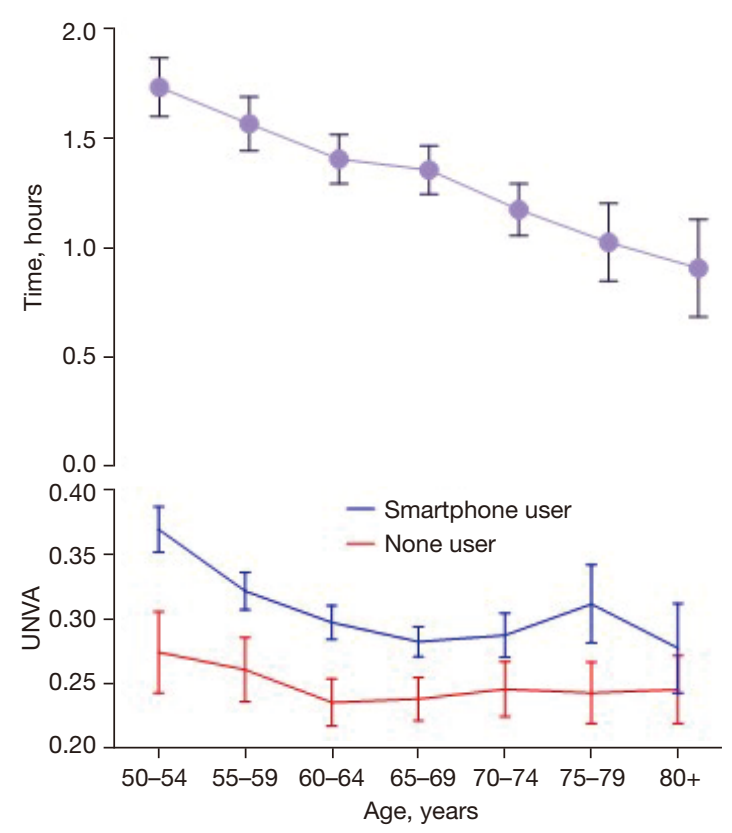

Figure 2 Time spend on smartphone and UNVA in urban population. UNVA, uncorrected near visual acuity.

and duration of smartphone use declined with age and UNVA, and the usage time was negatively correlated with visual quality. In this study, the overall adoption of smartphones among the elderly respondents was $67.7 \%$, which was slightly lower than that in the United States and the UK $(3,4)$. These results demonstrate that the use

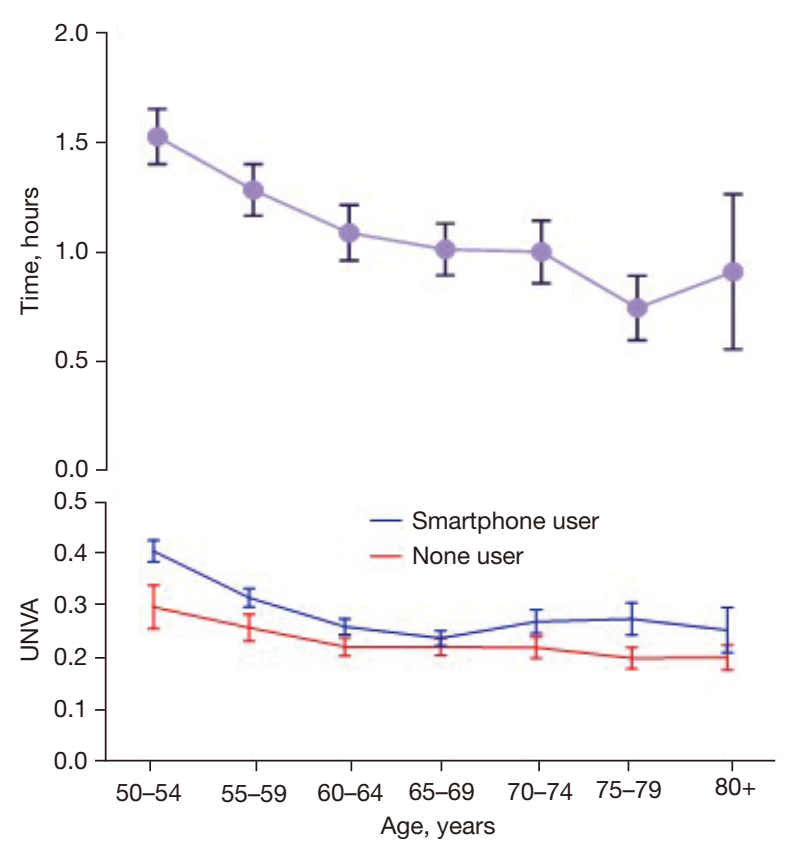

Figure 3 Time on smartphone and UNVA in rural population. UNVA, uncorrected near visual acuity.

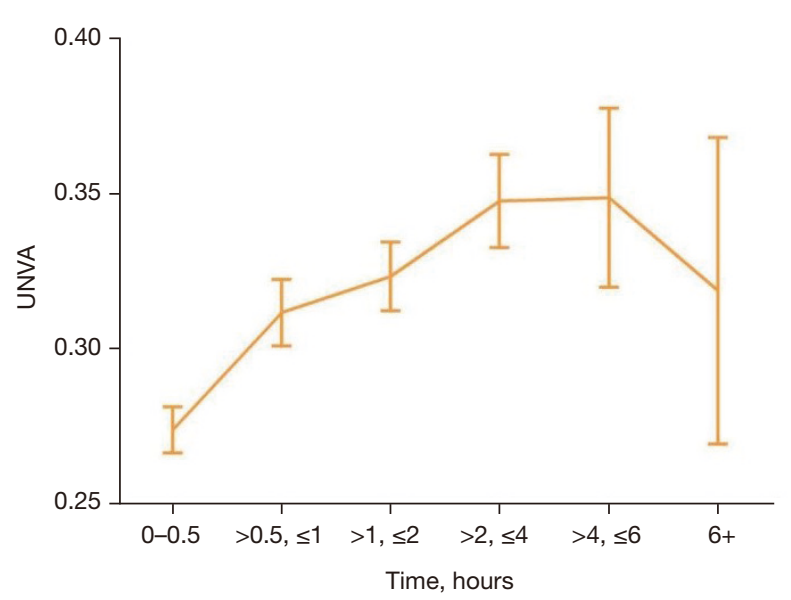

Figure 4 Trends between time on smartphone and UNVA. UNVA, uncorrected near visual acuity.

of smartphones varies in regions. Also, the popularity of phone use was decreased with age. In the 50-54-year age group, the adoption of phones was as high as $87.9 \%$, but for those aged $>80$ years, the adoption rate was only $32.3 \%$. The underlying reason for this may be that the younger subgroups had relatively good receptivity for intelligent applications and equipment, both physically and intellectually. Currently, cash circulation has drastically 


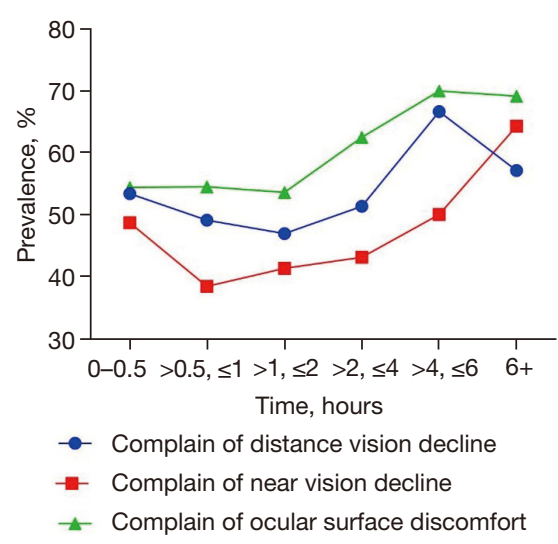

Figure 5 Rate of ocular complains in population by time on smartphone.

decreased in most places in China, which is due to increased mobile payments for public services, such as transportation, retail, and medical systems. These new patterns are causing problems for elderly individuals in several fields. Considering that unfamiliarity with smartphones may lead to predictable inconvenience in day-to-day life, the elderly are a vulnerable group who require special attention.

Generally, more than half of the respondents in this study spent less than 1 hour per day on smartphones, and a sharp decline in smartphone usage time with age was observed. Respondents who were able to use smartphones for a longer time usually had better UNVA reserves, which was mainly in the younger age groups. This result was obviously different from that of teenagers (5). According to a previous survey, teenagers could spend more than 4 hours per day on their smartphones (10). A possible explanation for this was that teenagers had better UNVA, and application designs were more suitable for them. Compared with the elderly, the changes in near vision caused by smartphones were transient in adolescents, and there was no step-down trend in UNVA. Another possible explanation was that, with ageing, presbyopia affected reading as well as any other near work, so they spent more time on other activities rather than their smartphones. However, in light of more social activities and information being displayed on smartphone platforms, this decline in smartphone usage time left older individuals vulnerable to online social connection.

In our study, UNVA decreased with age, which was consistent with the findings of previous studies. Interestingly, there was a plateau in UNVA from the age of 65 (lasting for approximately 15 years), following a dramatic decline from the age of 50 years. This trend has not been reported previously. The underlying reason for the phenomenon may be that some near work was necessary for many of the respondents during their work life before the age of 60-65 years, but after retirement, this burden disappeared; in addition, lens dilation at this age may lead to refractive changes; consequently, their UNVA stopped decreasing and remained fairly stable. Correspondingly, smartphone usage time shortened and became relatively fixed after the age of 60 years. The UNVA plateau provides us with an opportunity to rethink smartphone design for elderly users. Most smartphones were designed based on the potential premise of good near vision level (above 0.4 for everyone), while the UNVA of elderly people with smartphones maintained a presbyopia level around 0.3 . Also, from our investigation, smartphone owners had a higher average UNVA than non-smartphone owners, which suggested that UNVA affected smartphone usage. This near visual gap could result in considerable trouble in smartphone usage for the elderly. If the physiological trend of UNVA could not be changed in the elderly, we wonder whether the smartphone can lower the threshold of near vision for them. For the elderly, lowering the threshold of phone design to suit their low near vision allow more individuals to enjoy the convenience of smartphones.

The duration of electronic use affects vision quality (11), and previous studies have shown that prolonged phone use could lead to eye strain as well as smartphone addiction in teenagers (12). In our study, older people showed similar symptoms. With the prolonged use of smartphones, the discomfort of ocular surface gradually increased, combined with vision loss and reading difficulties. The rate of ocular surface discomfort rose sharply among respondents when the usage time was over 1-2 hours per day. This provided a reference for the eye comfort zone; that is, when the smartphone was used beyond a certain time period, there would be a surge in complaints. These results are essential for minimizing ocular discomfort in the elderly caused by smartphone designs.

The association between smartphone overuse and incidence of visual impairment had been studied in a systematic review and meta-analysis (7). In their study, the pooled results showed negative but not statistically significant associations between smartphone overuse and myopia, blurred vision in young adults. Similar to their study, older adults with smartphone overuse showed better near vision in the elderly in our research. As myopia patients generally have better near vision, these results also suggested a correlation between smartphone overuse and 
visual impairment in the elderly. However, our study did not analyze the subgroup of myopia, so further research is needed.

This study was carried out in both urban and rural areas. The rate of smartphone use in urban areas was higher than that in rural areas, which may be related to socioeconomic factors or living habits. The average UNVA were better among urban respondents than among rural respondents. However, complaints of ocular surface discomfort and vision decline were more frequent among urban respondents compared to rural respondents. Further investigation revealed that the urban group spent more time on smartphones than the rural group. The study confirmed that prolonged use of electronic products could decrease visual quality, and also suggested that eye habits might be an important factor in determining an individual's ocular comfort level, while good vision was not a guarantee of good vision quality.

The widespread use of smartphone was bound to be a significant challenge for the ageing population. Our investigation revealed two problems: the low level of smartphone usage among the elderly, and the problems caused by smartphones in the foreseeable future. It is believed that the elderly may be more receptive to various intelligent applications with the development of networks. Various channels could be created to help the elderly. For example, smartphone training classes for the elderly are very popular in China. Those who cannot handle smartphones and other intelligent products should be offered offline support. The public should have a plan to address such problems and keep a certain proportion of traditional channels, such as offline appointments for the elderly, so that this group will not be hindered by the digital barrier. On the other hand, how to create healthier smartphone designs is an ongoing issue. Intelligent applications of all kinds could be developed to address the visual needs, age, and feelings of the elderly.

This study had some limitations that should be considered. Firstly, the best correction of near vision for the participants was not addressed, as this research mainly focused on functional status instead of visual impairment. Also, as a relatively broad study, this research provided a rough estimate of visual status with smartphone usage but did not consider the details and potential mechanism, which require advanced study. Therefore, the surveys employed in this study might not accurately reflect the ocular health state. In the survey of smartphone usage time, as it was almost impossible to determine the exact time for individuals, a choice of interval rather than the precise time was provided, and thus, the subjective choice of the intervals may lead to bias.

In summary, this is the first study to reveal the relationship between smartphones and near vision in middle-aged and elderly people. Our study revealed the trends and regularities of smartphone usage with UNVA in the elderly. The near vision and smartphone usage showed a decrease by age in the study, and the level of near vision strongly limited the usage of smartphones. The results implied that smartphone usage was closely related to age and visual quality, which differed from adolescents. It is necessary to consider the demands of these individuals, according to their near vision level and age, which requires the guidance and endeavour of the public. As there are few similar studies, more rigorous and optimal research is needed in the future.

\section{Acknowledgments}

The authors thank all the Fujian Eye Study members for supporting the sampling and enumeration in this study, as well as the participants for their interest in this study.

Funding: This study was supported by the Natural Science Foundation of China (No. 81870672), Fujian Natural Science Foundation (No. 2019D007), and the China Postdoctoral Natural Science Foundation (No. 2019M662251).

\section{Footnote}

Reporting Checklist: The authors have completed the SURGE reporting checklist. Available at Available at https://apm.amegroups.com/article/view/10.21037/apm$21-3830 / \mathrm{rc}$

Data Sharing Statement: Available at https://apm.amegroups. com/article/view/10.21037/apm-21-3830/dss

Conflicts of Interest: All authors have completed the ICMJE uniform disclosure form (available at https://apm. amegroups.com/article/view/10.21037/apm-21-3830/coif). The authors have no conflicts of interest to declare.

Ethical Statement: The authors are accountable for all aspects of the work in ensuring that questions related to the accuracy or integrity of any part of the work are appropriately investigated and resolved. Approval for the 
study was granted by the Ethics Committee of Xiamen Eye Center affiliated with Xiamen University (approval No. XMYKZX-KY-2018-001). The study was conducted in accordance with the Declaration of Helsinki (as revised in 2013). Informed consent was obtained from all study participants.

Open Access Statement: This is an Open Access article distributed in accordance with the Creative Commons Attribution-NonCommercial-NoDerivs 4.0 International License (CC BY-NC-ND 4.0), which permits the noncommercial replication and distribution of the article with the strict proviso that no changes or edits are made and the original work is properly cited (including links to both the formal publication through the relevant DOI and the license). See: https://creativecommons.org/licenses/by-nc-nd/4.0/.

\section{References}

1. China Internet Network Information Center. 45th Statistical Report on Internet Development.2020. Available online: http://www.cac.gov.cn/2020-04/27/ c_1589535470378587.htm

2. Hew JJ. Hall of fame for mobile commerce and its applications: a bibliometric evaluation of a decade and a half (2000-2015). Telematics and Informatics 2017;34:43-66.

3. The Vision Council. Eyes overexposed: The digital device dilemma: digital eye strain report. 2016.

4. Office for National Statistics. Internet users in the UK 2017. 2017.

Cite this article as: Hu Q, Li Y, Wang B, Qin X, Ren T, Hu Y, $\mathrm{Li}$ X. The correlation between near vision and smartphone use among ageing populations. Ann Palliat Med 2022;11(2):560-567. doi: $10.21037 / \mathrm{apm}-21-3830$
5. Jaiswal S, Asper L, Long J, et al. Ocular and visual discomfort associated with smartphones, tablets and computers: what we do and do not know. Clin Exp Optom 2019;102:463-77.

6. Dirani M, Crowston JG, Wong TY. From reading books to increased smart device screen time. Br J Ophthalmol 2019;103:1-2.

7. Wang J, Li M, Zhu D, et al. Smartphone Overuse and Visual Impairment in Children and Young Adults: Systematic Review and Meta-Analysis. J Med Internet Res 2020;22:e21923.

8. Bourne RRA, Flaxman SR, Braithwaite T, et al. Magnitude, temporal trends, and projections of the global prevalence of blindness and distance and near vision impairment: a systematic review and meta-analysis. Lancet Glob Health 2017;5:e888-97.

9. World Population Prospects 2019. Population Division, Department of Economic and Social Affairs United Nations, 2019.

10. Kwon M, Kim DJ, Cho H, et al. The smartphone addiction scale: development and validation of a short version for adolescents. PLoS One 2013;8:e83558.

11. Tsubota K, Nakamori K. Dry eyes and video display terminals. N Engl J Med 1993;328:584.

12. Talens-Estarelles C, García-Marqués JV, Cervino A, et al. Use of digital displays and ocular surface alterations: A review. Ocul Surf 2021;19:252-65.

(English Language Editor: A. Kassem) 